\title{
DEFINICIÓN CONCEPTUAL DE LOS MEDIOS DE COMUNICACIÓN POR UN GRUPO DE JÓVENES ESPAÑOLES. EL VALOR DE INTERNET
}

\section{DEFINITION OF MASS MEDIA BY THE YOUNG PEOPLE. THE VALUE OF INTERNET}

\section{AUTOR/ES}

Ana Isabel Bernal Triviño: Doctora en Periodismo. Investigadora Grupo Labcom. Universidad de Málaga. Málaga (España).

anaisbernal2000@gmail.com

\section{RESUMEN}

El presente artículo analiza la percepción que los jóvenes españoles tienen de los medios de comunicación. El objetivo del estudio es conocer cómo los definen y concretar las fortalezas y debilidades de Internet frente al resto de los medios. Se ha utilizado una metodología cualitativa, con una muestra aleatoria formada por 57 alumnos de tercero de Periodismo de la Universidad de Málaga. Los resultados muestran que la prensa y la radio son medios más distantes para los jóvenes, a diferencia de la televisión e Internet. Sin embargo, la credibilidad de la televisión es peor que la radio y la prensa. En conclusión, los jóvenes valoran más Internet, y consideran que posee una mayor proyección.

\section{PALABRAS CLAVE}

Internet - Prensa - Radio - Televisión - Jóvenes - Valoración - Definición.

\section{ABSTRACT}

The present article analyzes the perception of the Spanish young people about the mass media. The objective of the study is how they define them and to make specific the strengths and weaknesses of Internet as opposed to the rest of mass media. Has been used a qualitative methodology, with a random sample formed by 57 students of third of Journalism of the University of Malaga. The results show that the press and the radio are media more distant for the young people, unlike the television and Internet. Nevertheless, the credibility of the television is worse than the radio and the press. In 
conciusion, the young people value more internet, ana they consiaer that it nas a greater projection.

\title{
KEY WORDS
}

Internet - Press - Radio - Television - Young people - Valuation - Definition.

\section{ÍNDICE}

\author{
․ 1. LOS JÓVENES, NUEVAS TECNOLOGÍAS Y MEDIOS DE \\ COMUNICACIÓN. ESTADO DE LA CUESTIÓN \\ 2. MATERIAL Y MÉTODOS \\ 2.1 La muestra \\ 2.2 La metodología \\ 3. RESULTADOS \\ 4. DISCUSIÓN Y CONCLUSIÓN \\ 5. BIBLIOGRAFÍA
}

\section{LOS JÓVENES, NUEVAS TECNOLOGÍAS Y MEDIOS DE COMUNICACIÓN. ESTADO DE LA CUESTIÓN}

La mayoría de los estudios sobre medios de comunicación y jóvenes en España se han centrado en aspectos vinculados a la violencia, las drogas, el sexo, su imagen en los medios, o su relación con las nuevas tecnologías (Injuve: 2008). Sobre estas últimas, destacan los trabajos europeos de Livingstone sobre niños, jóvenes e Internet, con especial interés en la seguridad y en los contenidos que consultan (Garmendia \& Garitaonandia: 2007). Junto a ellos destacan informes cuantitativos basados exclusivamente en el consumo de medios, tanto de la población adolescente como infantil (AIMC: 2008).

Al margen de estos estudios estadísticos que certifican que el consumo de Internet frente a otros medios de comunicación es superior entre los jóvenes (AIMC: 2007-2008) en comparación con edades superiores, no se han analizado nuevos campos, como la visión que la juventud tiene de los medios que consume. Esta área de análisis es importante porque los jóvenes están siendo los impulsores de cambios en el sistema mediático, donde ha predominado tradicionalmente el éxito de la televisión. Por ejemplo, se registran pérdidas de audiencia en la televisión, que se trasladan a Internet, en España, Europa y Estados Unidos (Bernal: 2005). No obstante, aunque en España existe una mayor penetración de la televisión sobre Internet (AIMC: 2007-2008) hay que considerar que la televisión es un medio extendido de forma casi hegemónica entre los hogares. Además, Internet exige una mayor implicación en su consumo, más activo (Nielsen, 2001), a diferencia de la televisión, que puede funcionar pero sin prestarle atención. 
Los jóvenes han nacido inmersos en la Sociedad de la Información. La llegada de las nuevas tecnologías ha propiciado una reconfiguración de las horas dedicadas al tiempo libre entre los jóvenes, ante la mayor oferta de ocio, centrada principalmente entre Internet, el teléfono móvil, los videojuegos y el resto de los medios de comunicación. Debido al uso preferente de estos nuevos soportes por los jóvenes, se les define como «screenagers» (Buckingham: 2001) adolescentes en constante relación con las tecnologías de la información y de la comunicación, generación Red (Cebrián: 2000), generación.com (AIMC: 2008) o generación @ (De Bofarull: 2005). Los productos informativos y de entretenimiento que los jóvenes demandan se caracterizan por unos rasgos que configuran nuevas formas de comunicación con las que se encuentran más identificados, como un acceso rápido a lo que quieren consultar, acción constante, la posibilidad de realizar dos actividades a la vez, la importancia de la imagen, la estructura hipertextual, que los soportes sean portátiles, y que fomenten su participación. Por lo tanto, buscan sentirse dueños en el proceso de consumo de las nuevas tecnologías (Bernal, 2009).

Sin embargo, se desconoce a largo plazo el alcance de Internet en el contexto del sistema mediático tras las experiencias de consumo que tiene la juventud. Según la regla de los 30 años de Saffo (Biagi: 1999), es necesario que transcurran tres décadas para que se consolide una nueva tecnología. Por lo tanto, como en el futuro los jóvenes determinarán el consumo de medios, es interesante conocer sus preferencias actuales para definir las tendencias del sistema comunicativo en los próximos años, en la medida en que cada medio de comunicación ofrece de una forma diferente su mensaje informativo.

\section{MATERIAL Y MÉTODOS}

\subsection{La muestra}

La muestra de este estudio se centra en la población juvenil. Sin embargo, existe cierta ambigüedad entre los autores para definir qué edades se integran en el concepto juventud. Para concretarlo, se siguió la recomendación de Naval y Sádaba (2005) y Vera (2005), quienes incluyen en este rango a aquellos que tienen una edad adulta, de entre 1920 años hasta los 30. Esta elección se ajustaba a los datos del informe Juventud en España (López Blasco: 2004), donde la lectura de noticias de actualidad era mayor a partir de los 20 años, sobre todo entre los universitarios. Por lo tanto, dentro de esta horquilla de edad, se optó por reducirla a quienes no hubiesen finalizado los estudios. Finalmente, se concluyó que los jóvenes que participasen en la prueba tuviesen entre 20 y 21 años. Son estudiantes que se encuentran en el ecuador de la carrera. Se seleccionó una muestra no probabilística aleatoria (Flick: 2004), formada por 54 alumnos de tercer curso de Periodismo de la Universidad de Málaga. La muestra era equitativa, compuesta por 27 mujeres y 27 hombres. La elección de esta muestra no tiene como fin generalizar los 
resultados, sino cumplir con el objetivo de la metodología cualitativa, donde prima la profundización sobre el hecho investigado.

\subsection{La metodología}

La pregunta que da origen a este artículo pertenece a una investigación cualitativa más amplia, cuya herramienta para la obtención de los datos ha sido una entrevista semiestructurada. El enfoque elegido responde a la necesidad de estudios de esta naturaleza en las Ciencias Sociales, como sostienen Livingstone (2007), Sierra (1999), o Valbuena (1997 ). Debido a las características del método inductivo, no es precisa la elaboración de hipótesis (Hernández, Fernández, \& Baptista: 2006). Los objetivos de este artículo son dos: que los alumnos definan a los medios de comunicación y comparar las fortalezas y debilidades de Internet frente al resto de los medios. Esta metodología permite analizar cómo perciben los jóvenes cada una de las formas de comunicación que ofrecen estos medios, y que expliquen y describan, con sus propias palabras, la percepción que tienen de los medios tras sus experiencias.

La prueba se realizó a los alumnos en el contexto de la propia universidad, en una de las aulas del centro que estaban vacías, para evitar que el alumno se distrajese y que pudiese concretarse en las preguntas. Las respuestas se identificaron por la letra E (de encuestado) y una enumeración asignada a cada uno, con el fin de mantener su anonimato.

La entrevista tenía 10 preguntas abiertas, donde se incluía la que origina este artículo: ¿cómo definirías, con pocas palabras, a cada uno de los medios de comunicación: prensa, revistas, radio, televisión e Internet? Al ser una pregunta de respuesta abierta, la codificación es más complicada. Sin embargo, esa dificultad se reduce por la propia naturaleza de la pregunta estructurada, que solicita al entrevistado que ofrezca una lista de ítems que se asemejan a las categorías de un investigación cualitativa (Hernández, Fernández, \& Baptista: 2006).

Una vez obtenidas las respuestas se procedió a la codificación, que se registró con el programa informático Atlas. No obstante, aunque la naturaleza de la metodología cualitativa no precisa la contabilización de los resultados, sí se evalúa la presencia de frecuencias para ver cuál de las definiciones es más utilizada para definir cada medio, como recomendaban Hernández, Fernández y Baptista (2006). La unidad de análisis, al ser una pregunta de estructura, son las palabras. Éstas se codificaron en un primer nivel en función de las categorías que ellos mismos ofrecieron, reduciéndose en aquellos casos en los que aplicaban sinónimos.

Las categorías se agruparon en función de cada medio de comunicación:

-Prensa: seriedad, credibilidad, antigüedad, aburrido, elitista, cultural, partidista, frío. 
-Revista: llamativa, entretenida, moderna, sensacionalismo, especialización.

-Radio: compañía, actualidad, entretenimiento, credibilidad, fácil de usar.

-Televisión: espectáculo, entretenimiento, poca credibilidad, vulgar, pasivo, atractivo, compañía, hipnotizadora, imagen, color, sonido.

-Internet: inmediatez, cómodo, moderno, interactividad, diseño atractivo, claro, textos concisos, futuro, nuevo medio, universal, sin límites.

Después se realiza una segunda codificación, que permite establecer vínculos entre los medios de comunicación. Esta clasificación se estructura en cinco puntos.

-Valores. Aquellas definiciones que destacan la cualidad o utilidad del medio, o el significado que representa para el sujeto.

-Contenidos. Términos que se refieren a algún aspecto relacionado con el contenido que ofrece ese medio de comunicación.

-Diseño o soporte. Definiciones que hacen referencia a cuestiones sobre el aspecto formal del mensaje informativo, así como a las cualidades de su soporte.

-Función. Conceptos vinculados a funciones que, según el sujeto, puede desempeñar el medio de comunicación. Por su naturaleza y para evitar la reiteración, se excluye la función informativa, propia de cualquier medio de comunicación.

-Uso. Serán aquellas definiciones que mencionan el comportamiento que el sujeto tiene ante el medio de comunicación, o aquellas que surgen como producto de la experiencia de su utilización.

La finalidad de la investigación permite que exista una aplicabilidad de los resultados en los medios de comunicación, cuyos responsables obtienen una primera aproximación a los valores y características con las que los jóvenes definen cada medio. Para evaluar la validez de la investigación se siguieron las directrices marcadas por Hernández, Fernández y Samperi (2006). Entre otros aspectos, se buscó un clima de confianza para que los alumnos fuesen sinceros, y se anotó en un cuaderno de bitácora los hechos que ocurrieron durante el desarrollo de la investigación. Igualmente, se cuidó la aplicación de los procedimientos de la prueba. 


\section{RESULTADOS}

La primera codificación de los resultados aporta una visión general muy concreta sobre la valoración de cada medio:

La seriedad de la prensa ha sido el concepto más repetido por los jóvenes para definirla. Junto a él, también sostiene una amplia mayoría su credibilidad. Sin embargo, después hacen referencia a valores menos positivos como la antigüedad del medio, que les parece aburrido, en ocasiones dirigido sólo hacia una población elitista, por el coste, o demasiado cultural. Con escasa representación, algunos alumnos mencionan otros conceptos, como ser un medio partidista y frío.

Para definir a las revistas, los jóvenes han utilizado con una frecuencia muy parecida dos conceptos: llamativas y entretenidas. También se refieren a él como un medio moderno, aunque le critican, en pocas ocasiones, el sensacionalismo que existe en algunas de estas publicaciones y su elevado precio. Sobre los contenidos, la única definición empleada hace referencia a su especialización y, en cuanto a su diseño, hacen especial hincapié en la aportación del color. La palabra actualidad es la más usada para definir a la radio, seguida a distancia de otras consideraciones, como su poder de entretenimiento o su credibilidad. Una amplia mayoría de la muestra ha reconocido que para ellos la radio significa compañía. En cuanto al soporte, bastantes alumnos han destacado su accesibilidad.

Sobre la televisión optan por usar, por igual frecuencia, los términos espectáculo y entretenimiento. De estos conceptos derivan los juicios que expresan sobre los contenidos, que los consideran poco creíbles, y vulgares. No obstante, indican que aunque es un medio pasivo, es atractivo y reconocen que también desempeña una función de compañía e incluso hipnotizadora. Sobre su diseño muestran pocas referencias, y muy evidentes, como el concepto imagen, seguido de color y sonido.

Para la mayoría, Internet representa la inmediatez, y resaltan su fácil uso y la comodidad, por lo que lo definen como un medio práctico, moderno e innovador. Además, resaltan su cualidad interactiva. Consideran que tiene un diseño muy atractivo, claro y que los textos son concisos. Otros valores, con menor frecuencia, han sido que Internet es un medio nuevo que representa el futuro, que les permite acceder sin límite de tiempo y espacio, que es imprescindible y universal.

La segunda codificación permite comparar los resultados de cada uno de los medios de comunicación:

La prensa e Internet han sido los dos medios con más valores registrados. Sin embargo, los significados de cada uno varían notablemente. Mientras la prensa se percibe como un medio más alejado de la población, frío, serio, y antiguo; Internet es visto como un medio más adaptado a los nuevos tiempos, universal e innovador. Sobre la televisión, 
las definiciones aportadas se centran en la imagen que ofrece el medio, basada en el espectáculo. La radio está ligada a la actualidad, por lo que consideran que es un medio al que acuden cuando quieren actualizar cualquier información.

En relación a los contenidos, se percibe que para ellos la credibilidad es un aspecto ligado a la antigüedad del medio, como es el caso de la prensa y de la radio. Sin embargo, la televisión carece de ella. Una opinión vinculada a la valoración aportada por los alumnos, que percibían a la televisión como un espectáculo, incluso vulgar. El sensacionalismo de las revistas es otro de los aspectos indicados, por lo que tienden a relacionar este medio con la prensa del "corazón". No obstante, aún con menor frecuencia, reflejan la existencia de otro tipo de revistas cuando mencionan la especialización de contenidos. Por último, los contenidos de Internet no son definidos por su credibilidad o su calidad, sino por dos rasgos principales del medio: su interactividad e inmediatez.

Sobre el diseño, al margen de la radio (que carece de él), es llamativo que la prensa no ha sido definida en ningún momento por este rasgo. Por el contrario, sí lo han sido las revistas, y sobre todo Internet, del que ha destacado dos aspectos: su diseño en general y la brevedad de sus textos.

Existe una relación personal con los medios. Al margen de la función informativa, dos asumen otras funciones, como la radio y la televisión, que no reconocen en el caso de Internet. Por un lado, se establece una relación entre el medio y el sujeto al considerar que su presencia desempeña una compañía. Esto sólo ocurre con la televisión y la radio, siendo en el último caso la característica más destacada. Por otro lado, el aporte de entretenimiento $\mathrm{u}$ ocio se concreta con un fin evasivo, como en la televisión, de la que resaltan su capacidad de hipnotizar.

Respecto al uso, mientras mencionan la facilidad de uso de la radio y de Internet, perciben a la televisión como un medio pasivo. No hacen referencia a los medios en soporte papel

\section{DISCUSIÓN Y CONCLUSIÓN}

La valoración de los contenidos evidencia que la imagen de la televisión se ha degradado entre los jóvenes. A ello se une su consideración como medio pasivo. Por lo tanto, ahí puede radicar la pérdida de audiencia juvenil de este medio en los últimos años.

Las revistas tienen su mayor cauce de futuro si mantienen la calidad de la especialización, una cualidad que no se anuncia en ningún otro medio y que garantiza su aceptación entre los jóvenes. 
En cuanto a la facilidad de uso de los medios, es importante puntualizar que sólo lo mencionan en aquellos cuyo soporte es tecnológico, y que requiere una mayor destreza, mientras que no hacen referencia a los medios en soporte papel, como la prensa o las revistas.

Sobre la función de compañía que pueden ejercer, en el caso de Internet es posible que no actúe con ese fin por dos razones. La primera porque es precisa la acción del sujeto para que el medio funcione. Por otra, los medios que producen compañía son aquellos con componente sonoro, que pueden sustituir el sonido de la voz humana, de los que carece Internet (sin contar con la presencia de videos multimedia) y la prensa papel.

Se percibe una relación entre los consumos estadísticos registrados y el número de conceptos utilizados para definir a cada medio. Los medios que más usan, Internet y la televisión, son aquellos en los que han empleado más definiciones. Su mayor utilización permite una descripción más detallada en consonancia con su mayor grado de conocimiento. Por el contrario, aquellos medios que registran una audiencia menor de la población juvenil, como la radio o la prensa, han sido los menos descritos.

Desde un punto de vista temporal, la prensa es el único medio que encuentran más alejado, al considerarlo antiguo, además de ser, según ellos, el más distanciado de la población ya que lo restringen a las clases sociales más altas. Por lo tanto, se identifican escasamente con él. Sin embargo, otros medios adquieren un valor más actual, como cuando consideran que las revistas o Internet son medios modernos. Una reflexión llamativa porque las revistas, a pesar de ser un medio en soporte papel, a diferencia de la prensa, sí tienen una mayor aceptación, en parte por la especialización de sus contenidos, con los que pueden identificarse según sus preferencias. No obstante, Internet es el único que registra una perspectiva prospectiva, ya que insisten en que es nuevo, universal y que representa el futuro de los medios de comunicación.

Los contenidos de Internet, al ser definidos por sus características, desvelan que los jóvenes no discuten la credibilidad de las informaciones que consultan, una respuesta destacada cuando desde diversos sectores se cuestiona la seguridad de los contenidos de la red.

En conclusión, las fortalezas de Internet radican en el diseño, porque se detecta un interés visual por la lectura en el nuevo medio, en la facilidad de uso y en la proyección que se percibe de él. Internet es el único medio en el que han reflejado una mayor identificación de nuevas formas de comunicación, adaptadas a las tecnologías y con mayor capacidad de futuro. Sus debilidades pueden estar en no cumplir con las expectativas mencionadas del medio: escasa actualización, diseño pobre, o menor credibilidad, y que no es capaz de generar una función personal, aunque quizás los jóvenes pueden imponer una nueva forma de relación con los medios, donde la compañía se sustituya por la utilidad inmediata. Sí debería de potenciar una mayor 
vinculación con los sujetos, de manera que lleguen a formar parte de su rutina y de su ámbito más cercano.

Este artículo constituye una primera aproximación a la consideración de los medios de comunicación por parte de los jóvenes. Por lo tanto, es un estudio que abre paso a nuevas investigaciones sobre esta temática que puedan delimitar desde otros enfoques metodológicos, como el cuantitativo o experimental, la aceptación de los medios desde diversos campos, como los contenidos, su portabilidad, o el diseño.

\section{BIBLIOGRAFÍA}

- AIMC (2008): Audiencia infantil/juvenil en medios en España 2008. Madrid, Asociación para la investigación de medios de comunicación, URL: www.aimc.es/09notas/ninos08.pdf, consulta: 9 de diciembre de 2008

- AIMC (2007-2008): EGM. Madrid, Asociación para la Investigación de Medios de Comunicación, URL: www.aimc.es/02egm/resumengm108.pdf, consulta: 8 de marzo de 2009

- BERNAL, A.I. (2009): Los nuevos medios de comunicación y los jóvenes. Madrid, Euroeditions, Fundación Europea para la Sociedad de la Información y la Administración Electrónica

- BERNAL, A.I. (2005): Situación de los estudios e investigaciones relacionadas con el periodismo en Internet y las nuevas tecnologías de la comunicación. Aproximación a la comunicación periodística en Internet. (Dir. Cabrera González, M.A.) Málaga, Universidad de Málaga. [tesina]

- BIAGI, S.(1999): Impacto de los medios, Méjico, International Thomson.

- BUCKINGHAM, D.(2001): Crecer en la era de los medios electrónicos: tras la muerte de la infancia. Madrid, Paideia.

- CEBRIÁN, J. (2000): La Red. Madrid, Suma de Letras.

- DE BOFARUlL, I. (2005): “Ocio en los medios de comunicación”, en Revista Juventud, $\mathrm{n}^{\mathrm{o}}$ 68, Madrid, Instituto de la Juventud, pp.116-127.

- FLICK, U. (2004): Introducción a la investigación cualitativa, Madrid, Fundación PaideiabGaliza. 
- GARMENDIA, M., y GARITAONANDIA, C. (2007): “Sonia Livingstone: Investigadora y experta internacional en Internet y niños y jóvenes", Revista Telos, $\mathrm{n}^{\mathrm{o}}$ 73. Madrid, Fundación Telefónica, URL: www.campusred.net $/$ telos / articulocuaderno.asp?idarticulo $=3 \&$ rev $=73$, consulta: 14 de abril de 2008.

- HERnÁNDEZ, R., FERNÁNDEZ, C., \& BAPTISTA, P. (2006), Metodología de la investigación, Madrid, Mc Graw Hill.

- INJUVE (2008): "Estudios e investigaciones Injuve", Madrid, Instituto de la Juventud, URL:

$\underline{\text { www.injuve.mtas.es/injuve/contenidos.type.action?type }=1579146 \& \text { menuId }=15}$ 79146\&mimenu=Estudios\%20e\%20Investigaciones, consulta: 9 de diciembre de 2008.

- LIVINGSTONE, S. (2007). “Los niños en Europa. Evaluación de los riesgos de Internet", en Revista Telos, n73, Madrid, Fundación Telefónica, URL:

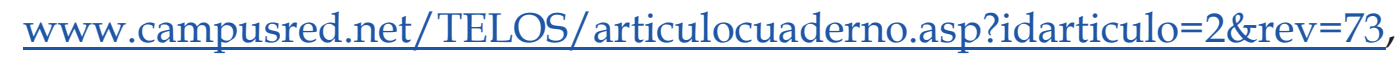
consulta: 8 de marzo de 2008.

- LÓPEZ BLASCO, A. ET ALT. (2005): Informe Juventud en España 2004, Madrid, Instituto de la Juventud, URL: www.injuve.mtas.es/injuve/contenidos.item, consulta: 8 de marzo de 2005.

- NAVAL, C. y SÁDABA, CH. (2005): Juventud y medios de comunicación frente a frente, en Revista Juventud, $\mathrm{n}^{\circ}$ 68, Madrid, Instituto de la Juventud, URL: www.injuve.mtas.es/injuve/contenidos.downloadatt.action?id=1836488182, consulta: 6 de enero de 2006.

- NIELSEN, J. (2001): Usabilidad. Diseño de sitios Web, Madrid, Prentice Hall.

- SIERRA, R. (1999): Tesis doctorales y trabajos de investigación científica, Madrid, Paraninfo.

- VAlBUENA DE LA FUENTE, F. (1997 ): Teoría de los usos y gratificaciones, Madrid, Noesis. 
- VERA, Julio. (2005): Medios de comunicación y socialización juvenil. Revista Juventud, $\mathrm{n}^{\circ}$ 68, Madrid. Instituto de la Juventud, URL:

(www.injuve.mtas.es/injuve/contenidos.downloadatt.action?id=1590639627, consulta: 6 de enero de 2006. 